H. UEDA

KODAI MATH. J.

17 (1994), 329-340

\title{
SOME ESTIMATES FOR MEROMORPHIC FUNCTIONS SHARING FOUR VALUES
}

Dedicated to Professor Nobuyuki Suita on the occasion of his 60th birthday

\author{
BY HideHARU UEDA
}

\section{Introduction}

In this paper the term "meromorphic function" will mean a meromorphic function in $\boldsymbol{C}$. We will use the standard notations of Nevanlinna theory: $T(r, f)$, $m(r, c, f), N(r, c, f), \bar{N}(r, c, f), N_{k}(r, c, f), \bar{N}_{k}(r, c, f)(c \in \boldsymbol{C} \cup\{\infty\}, k=1,2, \cdots)$, and we assume that the reader is familiar with the basic results in Nevanlinna theory as found in [3]. Further, we will use the notations and terminology defined in the following (i)-(vi):

(i) Let $f$ and $g$ be distinct nonconstant meromorphic functions. For $r>0$, put $T(r)=\max \{T(r, f), T(r, g)\}$. We write $\sigma(r)=S(r)$ for every function $\sigma:(0, \infty) \rightarrow(-\infty, \infty)$ satisfying $\sigma(r) / T(r) \rightarrow 0$ for $r \rightarrow \infty$ possibly outside a set of finite Lebesgue measure.

(ii) For two nonconstant meromorphic functions $f, g$ and $c \in \boldsymbol{C} \cup\{\infty\}$ we denote by $\bar{n}(r, c)=\bar{n}(r, c ; f, g)$ (resp. $\bar{n}_{1}(r, c)=\bar{n}_{1}(r, c ; f, g), \bar{n}_{3}(r, c)=\bar{n}_{3}(r, c ;$ $f, g)$ ) the number of distinct roots of at least one of the equations $f=c$ and $g=c$ in $|z| \leqq r$ (resp. the number of distinct common roots of $f=c$ and $g=c$ with the same multiplicities in $|z| \leqq r$, the number of distinct $c$-points of $f$ or $g$ which are not common to $f$ and $g$ in $|z| \leqq r)$. We write

$$
\begin{gathered}
\bar{N}(r, c)=\bar{N}(r, c ; f, g)=\int_{0}^{r}\{\bar{n}(t, c)-\bar{n}(0, c)\} / t d t+\bar{n}(0, c) \log r \\
\bar{N}_{j}(r, c)=\bar{N}_{j}(r, c ; f, g)=\int_{0}^{r}\left\{\bar{n}_{j}(t, c)-\bar{n}_{j}(0, c)\right\} / t d t+\bar{n}_{j}(0, c) \log r(j=1,3)
\end{gathered}
$$

and

$$
\bar{N}_{2}(r, c)=\bar{N}(r, c)-\bar{N}_{1}(r, c) .
$$

Further, for a complex number $a(\neq 0,1)$ we write

$$
\begin{aligned}
& \bar{N}(r)=\bar{N}(r, 0)+\bar{N}(r, 1)+\bar{N}(r, \infty)+\bar{N}(r, a), \\
& \bar{N}_{j}(r)=\bar{N}_{j}(r, 0)+\bar{N}_{j}(r, 1)+\bar{N}_{j}(r, \infty)+\bar{N}_{j}(r, a) \quad(j=1,2) .
\end{aligned}
$$

AMS classification 30D35

Received May 12, 1993. 
(iii) We say that $f$ and $g$ share the value $c I M^{\prime \prime}$ (resp. $C M^{\prime \prime}$ ) if $\bar{N}_{3}(r, c)$ $=S(r)\left(\right.$ resp. $\left.\bar{N}_{2}(r, c)=S(r)\right)$.

These notions $I M^{\prime \prime}$ and $C M^{\prime \prime}$ are slight generalizations of $I M$ (See [2, p 545].) and $C M$ (See [2, p 545].) (or " $C M$ " (See [5, p 172].), respectively. It is easily seen that Theorem F, Corollary 1, Lemma 2 in [2] and Theorem $\mathrm{B}^{\prime}$ in [5] remain valid if $I M$ and " $C M$ " are replaced by $I M$ " and $C M$ ", respectively. And so, using the argument of the proof of Theorem 1 in [5], we see that Theorems E and 2 in [2] are still true if $I M$ and $C M$ are replaced by $I M^{\prime \prime}$ and $C M^{\prime \prime}$, respectively. The function $\phi$ defined in $[4,5,7]$ satisfies $N(r, \infty, \phi)=S(r)$ and $m(r, \infty, \phi)=S(r)$ if $I M$ is replaced by $I M^{\prime \prime}$, and hence Lemma 2 in [7] remains true if $I M$ is replaced by $I M^{\prime \prime}$. (For convenience sake we state in $\S 3$ only a part of these facts without proof- which will be used to prove our results.)

(iv) For a given complex number $a(\neq 0,1)$ and two nonconstant meromorphic functions $f$ and $g$ we write

$$
\begin{aligned}
& n^{(k)}(r, c)=\#\left\{z_{c} \in C ;\left|z_{c}\right| \leqq r, z_{c} \text { is a } c \text {-point with multiplicity } p\right. \text { for } \\
& f \text { and with multiplicity } q \text { for } g \text {, where } p \text { and } q \text { satisfy } \\
& \left.\max (p, q) \geqq k+1 . z_{c} \text { is counted } \max (p, q)-k \text { times. }\right\} \\
& (c=0,1, \infty, a ; k=0,1,2), \text { and } \\
& N^{(k)}(r, c)=\int_{0}^{r}\left\{n^{(k)}(t, c)-n^{(k)}(0, c)\right\} / t d t+n^{(k)}(0, c) \log r \\
& (c=0,1, \infty, a ; k=0,1,2) .
\end{aligned}
$$

(v) Let $\bar{N}(r, f=g=c$ with $(p, q))$ denote the counting function of the $c$ points with multiplicity $p$ for $f$ and with multiplicity $q$ for $g$, each point counted once. Further, we denote such a $c$-point by $z_{c}(p, q)$.

(vi) Let $a(\neq 0,1)$ be a complex number, and let $f$ and $g$ be two nonconstant meromorphic functions. For $c=0,1, a, \infty$ we use the following notation:

$$
\begin{aligned}
& \bar{n}_{11}(r, c)=\#\left\{z_{c} \in C ;\left|z_{c}\right| \leqq r, f\left(z_{c}\right)=g\left(z_{c}\right)=c, f^{\prime}\left(z_{c}\right)=-g^{\prime}\left(z_{c}\right) \neq 0,\right. \\
& \left.f^{\prime \prime}\left(z_{c}\right)=g^{\prime \prime}\left(z_{c}\right), f^{\prime \prime \prime}\left(z_{c}\right)=-g^{\prime \prime \prime}\left(z_{c}\right), 3 f^{\prime \prime}\left(z_{c}\right)+2 \kappa(c)\left(f^{\prime}\left(z_{c}\right)\right)^{2}=0\right\} \\
& (c=0,1, a) \text {, where } \\
& \kappa(c)=(a+1)(c-1)\{(a-2) c-a(a-1)\} / a^{2}(a-1) \\
& +(a-2) c\left\{(a+1) c-\left(a^{2}+1\right)\right\} / a(a-1)^{2} \\
& =\left\{\begin{array}{l}
(a+1) / a \quad(c=0) \\
(2-a) /(a-1) \quad(c=1), \\
(1-2 a) / a(a-1) \quad(c=a)
\end{array}\right. \\
& \bar{n}_{11}(r, \infty)=\#\left\{z_{\infty} \in C ;\left|z_{\infty}\right| \leqq r, f(z)=\alpha /\left(z-z_{\infty}\right)+(a+1) / 3+\beta\left(z-z_{\infty}\right)+\cdots,\right. \\
& \left.g(z)=-\alpha /\left(z-z_{\infty}\right)+(a+1) / 3-\beta\left(z-z_{\infty}\right)+\cdots, \alpha \neq 0\right\} \\
& \text { ( } \alpha \text { and } \beta \text { may depend on } z_{\infty} \text { ), }
\end{aligned}
$$




$$
\bar{N}_{11}(r, c)=\int_{0}^{r}\left\{\bar{n}_{11}(t, c)-\bar{n}_{11}(0, c)\right\} / t d t+\bar{n}_{11}(0, c) \log r .
$$

Further, we write

$$
\bar{N}_{11}(r)=\bar{N}_{11}(r, 0)+\bar{N}_{11}(r, 1)+\bar{N}_{11}(r, \infty)+\bar{N}_{11}(r, a) .
$$

\section{Results}

In this section we give some estimates for meromorphic functions sharing four values $I M^{\prime \prime}$. Without loss of generality we may assume that these four shared values are $0,1, \infty$ and $a$.

In Theorems 1-4 we assume that $f$ and $g$ are distinct nonconstant meromorphic functions sharing four values $0,1, \infty$ and $a I M^{\prime \prime}$. Further, we assume in Theorems 1-3 that $g$ is not any Möbius transformation of $f$.

The following Theorem 1 is a refinement of a well known theorem of $R$. Nevanlinna [6, p 122].

THEOREM 1. $2 T(r)+3 \bar{N}_{11}(r) \leqq 3 \bar{N}_{2}(r)+S(r)$.

Our Theorem 2 contains a corresponding result to an author's uniqueness theorem for meromorphic functions sharing three values $C M[9$, Theorem 2].

THEOREM 2. $2 T(r)+3\left\{\bar{N}_{11}(r, 1)+\bar{N}_{11}(r, a)\right\}+\left\{N^{(2)}(r, 1)+N^{(2)}(r, a)\right\}$

$$
\leqq 3\{\bar{N}(r, 0)+\bar{N}(r, \infty)\}+2\left\{\bar{N}_{2}(r, 0)+\bar{N}_{2}(r, \infty)\right\}+S(r) .
$$

Our Theorem 3 is a refinement of so called 2-2-Theorem of G. Gundersen [2].

THEOREM 3. (i) There exists a positive constant $K_{1}$ satisfying

$$
\begin{gathered}
2 T(r)+3 \bar{N}_{11}(r)+\left\{N^{(2)}(r, 1)+N^{(2)}(r, a)\right\} \\
\leqq \\
\leqq
\end{gathered}
$$

We may take $K_{1}=11$ if $a=-1$, and $K_{1}=17$ otherwise.

(ii) Particularly if $\bar{N}_{1}(r, 0)=\bar{N}_{11}(r, 0)+S(r)$ and $\bar{N}_{1}(r, \infty)=\bar{N}_{11}(r, \infty)+S(r)$ hold, then we have

$$
\begin{aligned}
& 2 T(r)+3\left\{\bar{N}_{11}(r, 1)+\bar{N}_{11}(r, a)\right\}+\left\{N^{(2)}(r, 1)+N^{(2)}(r, a)\right\} \\
& \quad \leqq(13 / 2)\left\{\bar{N}_{2}(r, 0)+\bar{N}_{2}(r, \infty)\right\}+S(r) .
\end{aligned}
$$

Finally, using a method of E. Mues [4], we prove

THEOREM 4. (i) There exist positive constants $K_{2}$ and $K_{3}$ satisfying

$$
T(r) \leqq K_{2}\left\{\bar{N}_{1}(r, 0)+\bar{N}_{1}(r, 1)+\bar{N}_{1}(r, a)\right\}+K_{3} \bar{N}(r, \infty)+S(r) .
$$


We may take $\left(K_{2}, K_{3}\right)=(11 / 2,19 / 2)$ if $a=-1,1 / 2$ or 2 , and $\left(K_{2}, K_{3}\right)=(10,17)$ otherwise.

(ii) Particularly if $\bar{N}(r, f=g=c$ with $(2,1))+\bar{N}(r, f=g=c$ with $(1,2))=$ $S(r)$ for $c=0,1$ and $a$, then

$$
T(r) \leqq \bar{N}_{1}(r, 0)+\bar{N}_{1}(r, 1)+\bar{N}_{1}(r, a)+2 \bar{N}(r, \infty)+S(r) .
$$

(iii) Assume that $\bar{N}_{2}(r, c, f)+\bar{N}_{2}(r, c, g)=S(r)$ for $c=0,1$ and $a$, and that for each $c=0,1$ and $a \bar{N}(r, f=g=c$ with $(2,1))=S(r)$ or $\bar{N}(r, f=g=c$ with

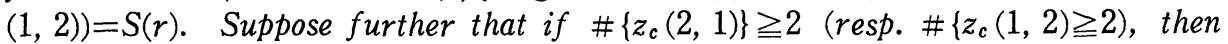
$g^{\prime}\left(z_{c}(2,1)\right)=$ const. $C_{c(2,1)}$ for all $z_{c}(2,1)$ 's $\left(\right.$ resp. $f^{\prime}\left(z_{c}(1,2)\right)$ (=const. $C_{c(1,2)}$ for all $z_{c}(1,2)$ 's). Then we have

$$
T(r) \leqq 2\left\{\bar{N}_{1}(r, 0)+\bar{N}_{1}(r, 1)+\bar{N}_{1}(r, a)+\bar{N}(r, \infty)\right\}+S(r) .
$$

Remark 1. The estimates of Theorems 1,2 and 3 (ii) are sharp in the case of $a=-\omega$, where $\omega(\neq 1)$ is a third root of 1 . Consider the two functions $F, G$ and two values $A, B$ in $[8, \mathrm{p} 94]$. If we put $f=F / A, g=G / A$, and $a=$ $B / A(=-\omega)$, then $f$ and $g$ are distinct meromorphic functions sharing four values $0,1, a, \infty I M$, and $g$ is not any Möbius transformation of $f$. Further, $f$ and $g$ satisfy the estimates of Theorems 1,2 and 3 (ii) with equality.

Remark 2. The estimate of Theorem 4 (ii) is sharp in the case of $a=$ $-1,1 / 2$ or 2 . For $a=-1$ this is illustrated by the pair of $F$ and $G$ in $[7$, Theorem 1]. For $a=1 / 2$ or 2 we can obtain an example from these $F$ and $G$ with the aid of a Möbius transformation.

Remark 3. The estimate of Theorem 4 (iii) is sharp in the case of $a=$ $-1 / 8,-8,1 / 9,9,8 / 9$ or $9 / 8$. For $a=-1 / 8$ this is illustrated by the pair of $f=\left(e^{z}+1\right) /\left(e^{z}-1\right)^{2}$ and $g=\left(e^{z}+1\right)^{2} / 8\left(e^{z}-1\right)$. This example is due to G. Gundersen [1]. For $a=-8,1 / 9,9,8 / 9$ or $9 / 8$ we can obtain an example from these $f$ and $g$ with the aid of a Möbius transformation.

\section{Lemmas}

In this section, we assume that $f$ and $g$ are distinct nonconstant meromorphic functions sharing four values $0,1, \infty, a I M^{\prime \prime}$. Then the following (3.1)

(3.8) hold :

$$
T(r, f)=T(r)+S(r), \quad T(r, g)=T(r)+S(r) ;
$$

(3.2) $\bar{N}(r)=2 T(r)+S(r)$;

$$
\begin{aligned}
& N(r, 0, f-g)=\bar{N}(r, 0, f-g)+S(r)=\bar{N}(r, 0)+\bar{N}(r, 1)+\bar{N}(r, a)+S(r) \\
& N^{(0)}(r, \infty)=N(r, \infty, f-g)+S(r) \\
& N(r, \infty, f)+N(r, \infty, g)=N^{(0)}(r, \infty)+\bar{N}(r, \infty)+S(r)
\end{aligned}
$$


(3.5) If $N_{0}\left(r, 0, f^{\prime}\right)$ refers only to those roots of $f^{\prime}=0$ such that $f \neq 0,1$ and $a$, and if $N_{0}\left(r, 0, g^{\prime}\right)$ is similarly defined, then $N_{0}\left(r, 0, f^{\prime}\right)=S(r)$ and $N_{0}(r, 0$, $\left.g^{\prime}\right)=S(r)$ (cf. [2, Lemma 2]);

(3.6) The function

$$
\phi_{5}=\frac{f^{\prime} g^{\prime}(f-g)^{2}}{f g(f-1)(g-1)(f-a)(g-a)}
$$

satisfies $T\left(r, \phi_{5}\right)=S(r)$ (cf. [4, p 113]);

$$
\begin{aligned}
& m\left(r, \infty, f^{\prime} /(f-g)\right)+N_{1}\left(r, \infty, f^{\prime} /(f-g)\right)=S(r), \\
& m\left(r, \infty, g^{\prime} /(g-f)\right)+N_{1}\left(r, \infty, g^{\prime} /(g-f)\right)=S(r) \quad(\text { cf. [7, Lemma 2]); }
\end{aligned}
$$

(3.8) Suppose further that $f$ and $g$ share two values of $0,1, \infty, a C M^{\prime \prime}$. Then $a=-1,1 / 2$ or 2 . In this case $f$ and $g$ is connected with exactly one of the following relations: $f+g \equiv 0(a=-1), f g \equiv 1(a=-1), f+g \equiv 1(a=1 / 2)$, $(f-1 / 2)(g-1 / 2) \equiv 1 / 4(a=1 / 2), f+g \equiv 2(a=2),(f-1)(g-1) \equiv 1(a=2) \quad(\mathrm{cf}$. $[5$, Theorem 1]).

For (3.1), (3.2) and the second equation of (3.4), see [4, Hilfssatz 1]. (3.3) and the first equation of (3.4) are easily verified by using the function $\psi$-which we denote by $\phi_{5}$ in this paper-in the proof of [4, Hilfssatz 1$]$.

\section{Proof of Theorems}

4.1. Proof of Theorem 1. Consider the functions

$$
\begin{gathered}
\phi_{1}=\frac{f^{\prime}(f-1)}{f(f-a)}-\frac{g^{\prime}(g-1)}{g(g-a)}, \\
\phi_{2}=\frac{f^{\prime}(f-a)}{f(f-1)}-\frac{g^{\prime}(g-a)}{g(g-1)}, \\
\phi_{3}=\frac{f^{\prime} f}{(f-1)(f-a)}-\frac{g^{\prime} g}{(g-1)(g-a)}, \\
\phi_{4}=\frac{f^{\prime}}{f(f-1)(f-a)}-\frac{g^{\prime}}{g(g-1)(g-a)} .
\end{gathered}
$$

If $\phi_{k} \equiv 0$ for $k=1,2,3$ or 4 , then it is easily seen that $f$ and $g$ share four values $0,1, a, \infty C M^{\prime \prime}$. Hence by (3.8) $g$ is a Möbius transformation of $f$, which contradicts our assumption.

Now, consider the case $\phi_{k} \neq \equiv$ for all $k=1,2,3,4$. We first note from the fundamental estimate of the logarithmic derivative and (3.1) that

$$
m\left(r, \phi_{k}\right)=S(r) \quad \text { for } \quad k=1,2,3,4 .
$$

By substituting into (4.1) the Taylor expansions of $f$ and $g$ at a $z_{1}(1,1)$ which 
is counted into $\bar{n}_{11}(r, 1)$, we see that $\phi_{1}$ has a zero whose multiplicity is at least four at this point. Hence from (4.1), (3.1), the first fundamental theorem and (4.5) it follows that

$$
\begin{aligned}
& \bar{N}(r, 1)+3 \bar{N}_{11}(r, 1) \leqq N\left(r, 0, \phi_{1}\right)+S(r) \leqq T\left(r, \phi_{1}\right)+S(r) \\
& \quad=N\left(r, \infty, \phi_{1}\right)+S(r) \leqq \bar{N}_{2}(r, 0)+\bar{N}_{2}(r, a)+\bar{N}_{2}(r, \infty)+S(r) .
\end{aligned}
$$

In the same way, we deduce from (4.2), (4.3) and (4.4) that

$$
\begin{aligned}
& \bar{N}(r, a)+3 \bar{N}_{11}(r, a) \leqq \bar{N}_{2}(r, 0)+N_{2}(r, 1)+\bar{N}_{2}(r, \infty)+S(r), \\
& \bar{N}(r, 0)+3 \bar{N}_{11}(r, 0) \leqq \bar{N}_{2}(r, 1)+\bar{N}_{2}(r, a)+\bar{N}_{2}(r, \infty)+S(r)
\end{aligned}
$$

and

$$
\bar{N}(r, \infty)+3 \bar{N}_{11}(r, \infty) \leqq \bar{N}_{2}(r, 0)+\bar{N}_{2}(r, 1)+\bar{N}_{2}(r, a)+S(r),
$$

respectively. Taking (3.2) into consideration, the combination of (4.6)-(4.9) yields the estimate of Theorem 1.

4.2. Proof of Theorem 2. Define $\phi,(j=1,2)$ by $(4 . j)$. From the argument of the first part of the proof of Theorem 1 we deduce that $\phi_{j} \neq 0(j=1,2)$. In this case, (4.6) and (4.7) hold, and so,

$$
\begin{gathered}
\bar{N}_{1}(r, 1)+\bar{N}_{1}(r, a)+3\left\{\bar{N}_{11}(r, 1)+\bar{N}_{11}(r, a)\right\} \\
\leqq 2\left\{\bar{N}_{2}(r, 0)+\bar{N}_{2}(r, \infty)\right\}+S(r) .
\end{gathered}
$$

Next, by (3.1), (3.2) and the first fundamental theorem

$$
\begin{gathered}
N(r, 1, f)+N(r, a, f)+N(r, 1, g)+N(r, a, g) \leqq 4 T(r)+S(r) \\
=2\{\bar{N}(r, 0)+\bar{N}(r, 1)+\bar{N}(r, a)+\bar{N}(r, \infty)\}+S(r) .
\end{gathered}
$$

Since $2\{\bar{N}(r, 1)+\bar{N}(r, a)\}+N^{(1)}(r, 1)+N^{(1)}(r, a) \leqq N(r, 1, f)+N(r, a, f)+N(r, 1$, $g)+N(r, a, g)+S(r)$ by the definition of $I M^{\prime \prime}$, it follows from (4.11) that

$$
N^{(1)}(r, 1)+N^{(1)}(r, a) \leqq 2\{\bar{N}(r, 0)+\bar{N}(r, \infty)\}+S(r) .
$$

Further, from (3.3) and the definition of $N^{(k)}(r, c)$ we deduce that

$$
\begin{gathered}
\bar{N}_{2}(r, 1)+\bar{N}_{2}(r, a)+N^{(2)}(r, 1)+N^{(2)}(r, a) \\
=N^{(1)}(r, 1)+N^{(1)}(r, a)+S(r) .
\end{gathered}
$$

Thus the combination of (3.2), (4.10), (4.13) and (4.12) yields the estimate of Theorem 2.

4.3.1. Proof of Theorem 3. (i) Define $\phi_{j}(j=1,2)$ by $(4 . j)$, and further define $\phi_{j}(j=5,6,7,8,9)$ as follows : 


$$
\begin{gathered}
\phi_{5}=\frac{f^{\prime} g^{\prime}(f-g)^{2}}{f g(f-1)(g-1)(f-a)(g-a)}, \\
\phi_{6}=\left\{\frac{f^{\prime \prime}}{f^{\prime}}-2 \frac{f^{\prime}}{f}-\frac{f^{\prime}}{f-1}-\frac{f^{\prime}}{f-a}\right\}-\left\{\frac{g^{\prime \prime}}{g^{\prime}}-2 \frac{g^{\prime}}{g}-\frac{g^{\prime}}{g-1}-\frac{g^{\prime}}{g-a}\right\}, \\
\phi_{7}=\left\{\frac{f^{\prime \prime}}{f^{\prime}}+2 \frac{f^{\prime}}{f}-\frac{f^{\prime}}{f-1}-\frac{f^{\prime}}{f-a}\right\}-\left\{\frac{g^{\prime \prime}}{g^{\prime}}+2 \frac{g^{\prime}}{g}-\frac{g^{\prime}}{g-1}-\frac{g^{\prime}}{g-a}\right\}, \\
\phi_{8}=\phi_{6}{ }^{2}-(1+a)^{2} \phi_{5}
\end{gathered}
$$

and

$$
\phi_{9}=\phi_{7}{ }^{2}-(1+a)^{2} \phi_{5} .
$$

Consider the case that $\phi_{j} \equiv 0$ for $\jmath=8$ or 9 . Then from (4.16), (4.17) and (3.6) we have $T\left(r, \phi_{\jmath}\right)=S(r)$ for $j=6$ or 7 . Noting that $N\left(r, \infty, \phi_{j}\right)=\bar{N}_{2}(r, 0)+$ $\bar{N}_{2}(r, \infty)+S(r)(\jmath=6,7)$ from (4.14), (4.15) and (3.5), we obtain $\bar{N}_{2}(r, 0)+\bar{N}_{2}(r, \infty)$ $=S(r)$, which implies that $g$ is a Möbius transformation of $f$ in view of (3.8).

Now, assume that $\phi_{j} \not \equiv 0$ for $j=8$ and 9. The substitution into (4.16) (resp. (4.17)) of the Taylor (resp. Laurent) expansions of $f$ and $g$ at a $z_{0}(1,1)$ (resp. $\left.z_{\infty}(1,1)\right)$ gives $\phi_{8}\left(z_{0}(1,1)\right)=0$ (resp. $\left.\phi_{9}\left(z_{\infty}(1,1)\right)=0\right)$. (See $[5$, pp. 174-175].) Especially if $z_{0}(1,1)$ (resp. $\left.z_{\infty}(1,1)\right)$ is counted into $\bar{n}_{11}(r, 0)$ (resp. $\bar{n}_{11}(r, \infty)$ ), then $\phi_{8}\left(\right.$ resp. $\left.\phi_{9}\right)$ has a zero whose multiplicity is at least two at this point. Hence, from (3.1), (3.3)-(3.6), the first fundamental theorem and the fundamental estimate of the logarithmic derivative it follows that

$$
\begin{gathered}
\bar{N}_{1}(r, 0)+\bar{N}_{11}(r, 0) \leqq N\left(r, 0, \phi_{8}\right)+S(r) \leqq T\left(r, \phi_{8}\right)+S(r) \\
=2 T\left(r, \phi_{6}\right)+S(r)=2\left\{\bar{N}_{2}(r, 0)+\bar{N}_{2}(r, \infty)\right\}+S(r)
\end{gathered}
$$

and

$$
\begin{gathered}
\bar{N}_{1}(r, \infty)+\bar{N}_{11}(r, \infty) \leqq N\left(r, 0, \phi_{9}\right)+S(r) \leqq T\left(r, \phi_{9}\right)+S(r) \\
=2 T\left(r, \phi_{7}\right)+S(r)=2\left\{\bar{N}_{2}(r, 0)+\bar{N}_{2}(r, \infty)\right\}+S(r) .
\end{gathered}
$$

Combining (4.18) and (4.19) with the estimate of Theorem 2, we have

$$
\begin{aligned}
& 2 T(r)+3 \bar{N}_{11}(r)+N^{(2)}(r, 1)+N^{(2)}(r, a) \\
& \leqq K_{1}\left\{\bar{N}_{2}(r, 0)+\bar{N}_{2}(r, \infty)\right\}+S(r)
\end{aligned}
$$

with $K_{1}=17$.

Finally, we consider the case $a=-1$. In this case we note that $\phi_{6}\left(z_{0}(1,1)\right)$ $=0$ and $\phi_{7}\left(z_{\infty}(1,1)\right)=0$ (Especially if $z_{0}(1,1)$ (resp. $\left.z_{\infty}(1,1)\right)$ is counted into $\bar{n}_{11}(r, 0)$ (resp. $\bar{n}_{11}(r, \infty)$ ), then $\phi_{6}\left(\right.$ resp. $\left.\phi_{7}\right)$ has a zero whose multiplicity is at least two at this point.), and so the above estimates (4.18) and (4.19) can be replaced by 


$$
\begin{gathered}
\bar{N}_{1}(r, 0)+\bar{N}_{11}(r, 0) \leqq N\left(r, 0, \phi_{6}\right)+S(r) \leqq T\left(r, \phi_{6}\right)+S(r) \\
=\bar{N}_{2}(r, 0)+\bar{N}_{2}(r, \infty)+S(r)
\end{gathered}
$$

and

$$
\begin{aligned}
& \bar{N}_{1}(r, \infty)+\bar{N}_{11}(r, \infty) \leqq N\left(r, 0, \phi_{7}\right)+S(r) \leqq T\left(r, \phi_{7}\right)+S(r) \\
& \quad=\bar{N}_{2}(r, 0)+\bar{N}_{2}(r, \infty)+S(r),
\end{aligned}
$$

respectively. Thus the combination of $(4.18)^{\prime},(4.19)^{\prime}$ and the estimate of Theorem 2 yields $(4.20)$ with $K_{1}=11$.

4.3.2. Proof of Theorem 3. (ii) Define $\phi_{10}$ and $\phi_{11}$ by

$$
\begin{aligned}
& \phi_{10}=\left\{\frac{f^{\prime \prime}}{f^{\prime}}+\frac{f^{\prime}}{f}-\frac{f^{\prime}}{f-1}-\frac{f^{\prime}}{f-a}\right\}-\left\{\frac{g^{\prime \prime}}{g^{\prime}}+\frac{g^{\prime}}{g}-\frac{g^{\prime}}{g-1}-\frac{g^{\prime}}{g-a}\right\}, \\
& \phi_{11}=\left\{\frac{f^{\prime \prime}}{f^{\prime}}-\frac{f^{\prime}}{f}-\frac{f^{\prime}}{f-1}-\frac{f^{\prime}}{f-a}\right\}-\left\{\frac{g^{\prime \prime}}{g^{\prime}}-\frac{g^{\prime}}{g}-\frac{g^{\prime}}{g-1}-\frac{g^{\prime}}{g-a}\right\},
\end{aligned}
$$

respectively. Assume first that $\phi_{10} \equiv 0$. In this case we have $f^{\prime} f /(f-1)(f-a)$ $\equiv L g^{\prime} g /(g-1)(g-a)$ with a nonzero constant $L$. If $L=1$, then $f$ and $g$ share four values $0,1, \infty$, a $C M^{\prime \prime}$, and so by (3.8) $g$ is a Möbius transformation of $f$. Unless $L=1$, then $\bar{N}(r, 0)=\bar{N}_{1}(r, 0)+S(r)=\bar{N}_{11}(r, 0)+S(r)=S(r)$ and $\bar{N}_{1}(r, 1)+$ $\bar{N}_{1}(r, a)+\bar{N}_{1}(r, \infty)=S(r)$. Applying Theorem $4(\mathrm{i})$-which will be proved laterto $1 / f$ and $1 / g$ with shared four values $\infty, 1,0,1 / a I M^{\prime \prime}$, we conclude that there does not exist such a pair of $f$ and $g$. The case of $\phi_{11} \equiv 0$ can be handled in the same way as the one of $\phi_{10} \equiv 0$.

Now, consider the case of $\phi_{10} \not \equiv 0$ and $\phi_{11} \not \equiv 0$. By substituting the Taylor (resp. Laurent) expansions of $f$ and $g$ at a $z_{0}(1,1)$ (resp. $z_{\infty}(1,1)$ ) which is counted into $\bar{n}_{11}(r, 0)$ (resp. $\bar{n}_{11}(r, \infty)$ ) into (4.21) (resp. (4.22)), we see that $\phi_{10}$ (resp. $\phi_{11}$ ) has a zero whose multiplicity is at least two at this point. Hence from (4.21) and (4.22) it follows that

and

$$
\begin{aligned}
2 \bar{N}_{1}(r, 0) & =2 \bar{N}_{11}(r, 0)+S(r) \leqq N\left(r, 0, \phi_{10}\right)+S(r) \\
& \leqq T\left(r, \phi_{10}\right)+S(r)=\bar{N}_{2}(r, 0)+S(r)
\end{aligned}
$$

$$
\begin{aligned}
2 \bar{N}_{1}(r, \infty) & =2 \bar{N}_{11}(r, \infty)+S(r) \leqq N\left(r, 0, \phi_{11}\right)+S(r) \\
& \leqq T\left(r, \phi_{11}\right)+S(r)=\bar{N}_{2}(r, \infty)+S(r),
\end{aligned}
$$

respectively. Combining these with the estimate of Theorem 2, we obtain the estimate of Theorem 3 (ii).

4.4.1. Proof of Theorem 4. (i) By (3.1) we easily see that $T\left(r, f^{\prime}\right)+$ $T\left(r, g^{\prime}\right) \leqq 2 T(r)+2 \bar{N}(r, \infty)+S(r)$, and from (3.3) and (3.5) it follows that $\bar{N}\left(r, 0, f^{\prime}\right)+\bar{N}\left(r, 0, g^{\prime}\right)=\bar{N}_{2}(r, 0)+\bar{N}_{2}(r, 1)+\bar{N}_{2}(r, a)+S(r)$. Hence using (3.2), we get 


$$
\begin{aligned}
& m\left(r, 0, f^{\prime}\right)+m\left(r, 0, g^{\prime}\right)+N_{1}\left(r, 0, f^{\prime}\right)+N_{1}\left(r, 0, g^{\prime}\right) \\
& \quad \leqq \bar{N}_{1}(r, 0)+\bar{N}_{1}(r, 1)+\bar{N}_{1}(r, a)+3 \bar{N}(r, \infty)+S(r) .
\end{aligned}
$$

Now, we define two auxiliary functions $\phi_{12}$ and $\phi_{13}$ :

$$
\begin{gathered}
\phi_{12}=2 \frac{f^{\prime \prime}}{f^{\prime}}-3\left\{\frac{f^{\prime}}{f}+\frac{f^{\prime}}{f-1}+\frac{f^{\prime}}{f-a}\right\}-2 \frac{g^{\prime \prime}}{g^{\prime}}+2\left\{\frac{g^{\prime}}{g}+\frac{g^{\prime}}{g-1}+\frac{g^{\prime}}{g-a}\right\} \\
+\frac{f^{\prime}-2 g^{\prime}}{f-g}
\end{gathered}
$$

and

$$
\begin{gathered}
\phi_{13}=2 \frac{g^{\prime \prime}}{g^{\prime}}-3\left\{\frac{g^{\prime}}{g}+\frac{g^{\prime}}{g-1}+\frac{g^{\prime}}{g-a}\right\}-2 \frac{f^{\prime \prime}}{f^{\prime}}+2\left\{\frac{f^{\prime}}{f}+\frac{f^{\prime}}{f-1}+\frac{f^{\prime}}{f-a}\right\} \\
+\frac{g^{\prime}-2 f^{\prime}}{g-f} .
\end{gathered}
$$

Making use of (3.3), (3.4), (3.5) and (3.7), we obtain the following estimates for $N\left(r, \infty, \phi_{12}\right)$ and $N\left(r, \infty, \phi_{13}\right)$ :

$$
\begin{aligned}
& \bar{N}_{1}(r, 0)+\bar{N}_{1}(r, 1)+\bar{N}_{1}(r, a)+\bar{N}_{2}(r, \infty)+\sum_{p \geqq 3} \bar{N}(r, f=g=0,1 \text { or } a \\
& \text { with }(p, 1))+S(r) \leqq N\left(r, \infty, \phi_{12}\right) \leqq \bar{N}_{1}(r, 0)+\bar{N}_{1}(r, 1)+\bar{N}_{1}(r, a) \\
& \quad+\bar{N}(r, \infty)+\sum_{p \geq 3} \bar{N}(r, f=g=0,1 \text { or } a \text { with }(p, 1))+S(r), \\
& \bar{N}_{1}(r, 0)+\bar{N}_{1}(r, 1)+\bar{N}_{1}(r, a)+\bar{N}_{2}(r, \infty)+\sum_{q \geq 3} \bar{N}(r, f=g=0,1 \text { or } a \\
& \text { with }(1, q))+S(r) \leqq N\left(r, \infty, \phi_{13}\right) \leqq \bar{N}_{1}(r, 0)+\bar{N}_{1}(r, 1)+\bar{N}_{1}(r, a) \\
& \quad+\bar{N}(r, \infty)+\sum_{q \geq 3} \bar{N}(r, f=g=0,1 \text { or } a \text { with }(1, q))+S(r) .
\end{aligned}
$$

According to E. Mues' calculations (See [4, pp. 116-117].) we have

$$
\begin{aligned}
& \left(\phi_{12}{ }^{2} / 2 \phi_{5}\right)\left(z_{0}(2,1)\right)=(a+1)^{2}, \quad\left(\phi_{13}{ }^{2} / 2 \phi_{5}\right)\left(z_{0}(1,2)\right)=(a+1)^{2}, \\
& \left(\phi_{12}{ }^{2} / 2 \phi_{5}\right)\left(z_{1}(2,1)\right)=(2-a)^{2}, \quad\left(\phi_{13}{ }^{2} / 2 \phi_{5}\right)\left(z_{1}(1,2)\right)=(2-a)^{2}, \\
& \left(\phi_{12}{ }^{2} / 2 \phi_{5}\right)\left(z_{a}(2,1)\right)=(2 a-1)^{2} \quad \text { and } \quad\left(\phi_{13}{ }^{2} / 2 \phi_{5}\right)\left(z_{a}(1,2)\right)=(2 a-1)^{2} .
\end{aligned}
$$

As will be shown later

$$
\phi_{12}^{2} / 2 \phi_{5} \not \equiv(a+1)^{2},(2-a)^{2},(2 a-1)^{2}
$$

and

$$
\phi_{13}{ }^{2} / 2 \phi_{5} \neq(a+1)^{2},(2-a)^{2},(2 a-1)^{2}
$$

hold. Hence from (3.6), (3.7), (4.26), (4.27) and (4.23) we deduce that 


$$
\begin{aligned}
& \bar{N}(r, f=g=0,1 \text { or } a \text { with }(2,1))+\bar{N}(r, f=g=0,1 \text { or } a \text { with } \\
& (1,2)) \leqq N\left(r,(a+1)^{2}, \phi_{12}{ }^{2} / 2 \phi_{5}\right)+N\left(r,(2-a)^{2}, \phi_{12}{ }^{2} / 2 \phi_{5}\right) \\
& +N\left(r,(2 a-1)^{2}, \phi_{12}{ }^{2} / 2 \phi_{5}\right)+N\left(r,(a+1)^{2}, \phi_{13}{ }^{2} / 2 \phi_{5}\right) \\
& +N\left(r,(2-a)^{2}, \phi_{13}{ }^{2} / 2 \phi_{5}\right)+N\left(r,(2 a-1)^{2}, \phi_{13}{ }^{2} / 2 \phi_{5}\right) \\
& \leqq 6\left\{N\left(r, \infty, \phi_{12}\right)+N\left(r, \infty, \phi_{13}\right)\right\}+S(r) \\
& \leqq 12\left\{\bar{N}_{1}(r, 0)+\bar{N}_{1}(r, 1)+\bar{N}_{1}(r, a)+\bar{N}(r, \infty)\right\} \\
& +6 \sum_{p \geq 3} \bar{N}(r, f=g=0,1 \text { or } a \text { with }(p, 1)) \\
& +6 \sum_{q \geq 3} \bar{N}(r, f=g=0,1 \text { or } a \text { with }(1, q))+S(r) \\
& \leqq 18\left\{\bar{N}_{1}(r, 0)+\bar{N}_{1}(r, 1)+\bar{N}_{1}(r, a)\right\}+30 \bar{N}(r, \infty)+S(r) .
\end{aligned}
$$

Together with (3.2) and (4.23), this yields

$$
\begin{aligned}
& 2 T(r)=\bar{N}(r)+S(r)=\left\{\bar{N}_{1}(r, 0)+\bar{N}_{1}(r, 1)+\bar{N}_{1}(r, a)\right\} \\
& +\left\{\bar{N}_{2}(r, 0)+\bar{N}_{2}(r, 1)+\bar{N}_{2}(r, a)\right\}+\bar{N}(r, \infty)+S(r) \\
& \leqq\left\{\bar{N}_{1}(r, 0)+\bar{N}_{1}(r, 1)+\bar{N}_{1}(r, a)\right\}+\bar{N}(r, f=g=0,1 \text { or } a \text { with }(2,1)) \\
& +\bar{N}(r, f=g=0,1 \text { or } a \text { with }(1,2))+N_{1}\left(r, 0, f^{\prime}\right)+N_{1}\left(r, 0, g^{\prime}\right)+ \\
& \bar{N}(r, \infty)+S(r) \leqq 20\left\{\bar{N}_{1}(r, 0)+\bar{N}_{1}(r, 1)+\bar{N}_{1}(r, a)\right\}+34 \bar{N}(r, \infty)+S(r) .
\end{aligned}
$$

This gives the estimate of Theorem 4 (i) with $\left(K_{2}, K_{3}\right)=(10,17)$.

Consider the case of $a=-1$. Then, $a+1=0$ and $(2-a)^{2}=(2 a-1)^{2}$ hold, so that (4.30) is replaced by

$$
\begin{aligned}
\bar{N}(r, f & =g=0,1 \text { or } a \text { with }(2,1))+\bar{N}(r, f=g=0,1 \text { or } a \text { with }(1,2)) \\
& \leqq 3\left\{N\left(r, \infty, \phi_{12}\right)+N\left(r, \infty, \phi_{13}\right)\right\}+S(r) \\
& \leqq 9\left\{\bar{N}_{1}(r, 0)+\bar{N}_{1}(r, 1)+\bar{N}_{1}(r, a)\right\}+15 \bar{N}(r, \infty)+S(r) .
\end{aligned}
$$

Combining this with (3.2) and (4.23), we obtain the estimate of Theorem 4(i) with $\left(K_{2}, K_{3}\right)=(11 / 2,19 / 2)$. The cases of $a=1 / 2$ and 2 can be handled in the same way.

It remains to show (4.28) and (4.29). We prove only that $\phi_{12}{ }^{2} / 2 \phi_{5} \not \equiv(a+1)^{2}$ since the other cases can be handled in the same way. We assume that $\phi_{12}{ }^{2} \equiv$ $2(a+1)^{2} \phi_{5}$, and will seek a contradiction. Consider first the case of $a \neq-1$. By the symmetry of $\phi_{5}$ on $f$ and $g$ we have

$$
\phi_{12}{ }^{2} \equiv 2(a+1)^{2} \phi_{5} \equiv \phi_{13}{ }^{2} \text {. }
$$

If $\bar{N}_{1}(r, \infty) \neq S(r)$, then by $(3.4)$ there exists a $z_{\infty}(1,1)$ satisfying $\operatorname{Res}\left(z_{\infty}(1,1)\right.$, 
$f) \neq \operatorname{Res}\left(z_{\infty}(1,1), g\right)$. Let $\alpha=\operatorname{Res}\left(z_{\infty}(1,1), f\right)$ and $\alpha^{\prime}=\operatorname{Res}\left(z_{\infty}(1,1), g\right)$. Simple computations on (4.31) give $\left\{\left(2 \alpha-\alpha^{\prime}\right) /\left(\alpha-\alpha^{\prime}\right)\right\}^{2}=0=\left\{\left(2 \alpha^{\prime}-\alpha\right) /\left(\alpha^{\prime}-\alpha\right)\right\}^{2}$, which is impossible. Hence $\bar{N}_{1}(r, \infty)=S(r)$, so that equality (up to an $S(r)$ term) must hold everywhere in (4.26) and (4.27), and further by (3.6) and (4.31) all sides of (4.26) and (4.27) are equal to $S(r)$. Together with (4.23) this yields

and

$$
\begin{aligned}
& m(r, 0, f)+m(r, 1, f)+m(r, a, f)+m(r, 0, g) \\
& \quad+m(r, 1, g)+m(r, a, g)=S(r) \\
& N_{1}\left(r, 0, f^{\prime}\right)+N_{1}\left(r, 0, g^{\prime}\right)=S(r)
\end{aligned}
$$

$$
\bar{N}_{1}(r, 0)+\bar{N}_{1}(r, 1)+\bar{N}_{1}(r, a)=S(r),
$$

so that for $c=0,1$ and $a$ we have

$$
\begin{aligned}
T(r)= & T(r, 1 /(f-c))+S(r)=m(r, c, f)+N(r, c, f)+S(r) \\
\leqq & m(r, c, f)+\bar{N}_{1}(r, c)+2 \bar{N}(r, f=g=c \text { with }(2,1)) \\
& +\bar{N}(r, f=g=c \text { with }(1,2))+3 N_{1}\left(r, 0, f^{\prime}\right)+3 N_{1}\left(r, 0, g^{\prime}\right)+S(r) \\
= & 2 \bar{N}(r, f=g=c \text { with }(2,1))+\bar{N}(r, f=g=c \text { with }(1,2))+S(r) \\
\leqq & 2 \bar{N}(r, f=g=c \text { with }(2,1))+N(r, c, g) / 2+S(r) \\
\leqq & 2 \bar{N}(r, f=g=c \text { with }(2,1))+T(r) / 2+S(r) .
\end{aligned}
$$

(4.32) guarantees the existence of all of $z_{0}(2,1), z_{1}(2,1)$ and $z_{a}(2,1)$ (cf. [4, p. 116]), which implies that $(a+1)^{2}=(2-a)^{2}=(2 a-1)^{2}$. This is impossible. Next, consider the case of $a=-1 . \phi_{12} \equiv 0$ yields $f^{\prime} / f+f^{\prime} /(f-1)+f^{\prime} /(f+1)-\left(f^{\prime}-2 g^{\prime}\right) /$ $(f-g)+g^{\prime} / g+g^{\prime} /(g-1)+g^{\prime} /(g+1)-\left(g^{\prime}-2 f^{\prime}\right) /(g-f) \equiv 0$, i.e.,

$$
(f-g)^{3} / f(f-1)(f+1) g(g-1)(g+1) \equiv A
$$

with a nonzero constant $A$. If $\bar{N}_{1}(r, \infty) \neq S(r)$, then by (3.4) there exists a $z_{\infty}(1,1)$ satisfying $\operatorname{Res}\left(z_{\infty}(1,1), f\right) \neq \operatorname{Res}\left(z_{\infty}(1,1), g\right)$. By substituting the Laurent expansions of $f$ and $g$ at such a point $z_{\infty}(1,1)$ into (4.33) we obtain $A=0$, which is a contradiction. Hence $\bar{N}_{1}(r, \infty)=S(r)$, and so using the same argument as in the case of $a \neq-1$, we arrive at a contradiction.

This completes the proof of Theorem 4 (i).

4.4.2. Proof of Theorem 4. (ii) From our assumption and (4.23) we have $\bar{N}_{2}(r, 0)+\bar{N}_{2}(r, 1)+\bar{N}_{2}(r, a)=\bar{N}\left(r, 0, f^{\prime}\right)+\bar{N}\left(r, 0, g^{\prime}\right)+S(r)=\bar{N}_{1}\left(r, 0, f^{\prime}\right)+$ $\bar{N}_{1}\left(r, 0, g^{\prime}\right)+S(r) \leqq \bar{N}_{1}(r, 0)+\bar{N}_{1}(r, 1)+\bar{N}_{1}(r, a)+3 \bar{N}(r, \infty)+S(r)$. Hence by $(3.2)$ $2 T(r) \leqq 2\left\{\bar{N}_{1}(r, 0)+\bar{N}_{1}(r, 1)+\bar{N}_{1}(r, a)\right\}+4 \bar{N}(r, \infty)+S(r)$.

4.4.3. Proof of Theorem 4. (iii) We make use of the proof of Theorem 4 (i ). Simple calculations give $\phi_{12}\left(z_{0}(2,1)\right)=-2 g^{\prime}\left(z_{0}(2,1)\right)(1+1 / a), \phi_{12}\left(z_{1}(2,1)\right)=$ 
$2 g^{\prime}\left(z_{1}(2,1)\right)\{1+1 /(1-a)\}, \phi_{12}\left(z_{a}(2,1)\right)=2 g^{\prime}\left(z_{a}(2,1)\right)\{1 / a+1 /(a-1)\}, \phi_{13}\left(z_{0}(1,2)\right)=$ $-2 f^{\prime}\left(z_{0}(1,2)\right)(1+1 / a), \quad \phi_{13}\left(z_{1}(1,2)\right)=2 f^{\prime}\left(z_{1}(1,2)\right)\{1+1 /(1-a)\}$ and $\phi_{13}\left(z_{a}(1,2)\right)=$ $2 f^{\prime}\left(z_{a}(1,2)\right)\{1 / a+1 /(a-1)\}$. Hence, if neither $\phi_{12}$ nor $\phi_{13}$ is constant, using our assumptions the above estimate (4.30) can be replaced by

$$
\begin{aligned}
& \vec{N}(r, f=g=0,1 \text { or } a \text { with }(2,1))+\bar{N}(r, f=g=0,1 \text { or } a \\
& \text { with }(1,2)) \leqq 3\left\{\bar{N}_{1}(r, 0)+\bar{N}_{1}(r, 0)+\bar{N}_{1}(r, 1)+\bar{N}_{1}(r, a)+\bar{N}(r, \infty)\right\} .
\end{aligned}
$$

Further, under our assumption, $\bar{N}_{2}(r, 0)+\bar{N}_{2}(r, 1)+\bar{N}_{2}(r, a)=\bar{N}(r, f=g=0,1$ or $a$ with $(2,1))+\bar{N}(r, f=g=0,1$ or $a$ with $(1,2))+S(r)$ holds. Thus the estimate of Theorem 4(iii) follows from $(4.30)^{\prime}$ and (3.2). It remains to consider the case that $\phi_{12}$ or $\phi_{13}$ is constant. In each case, we easily obtain $(f-g)^{3} /$ $\{f g(f-1)(g-1)(f-a)(g-a)\} \equiv e^{A z+B}$ with two constants $A$ and $B$. This implies that $\bar{N}_{1}(r, 0)+\bar{N}_{1}(r, 1)+\bar{N}_{1}(r, a)+\bar{N}(r, \infty)=S(r)$. But by Theorem 4 (i) there does not exist such a pair of $f$ and $g$.

Acknowledgement. The author is very grateful to the referee for valuable comments.

\section{REFERENCES}

[1] Gundersen, G.G., Meromorphic functions that share three or four values, J. London Math. Soc., 20 (1979), 457-465.

[2] Gundersen, G.G., Meromorphic functions that share four values, Trans. Amer. Math. Soc., 277 No. 2 (1983), 545-567.

[3] Hayman, W.K., Meromorphic Functions, Clarendon Press, Oxford, 1964.

[4] Mues, E., Bemerkungen zum Vier-Punkte-Satz, Complex Methods on Partial Differential Equation, Mathematical Research, 53, Akademie-Verlag, Berlin, 1989, 109-117.

[5] Mues, E., Meromorphic functions sharing four values, Complex Variables Theory Appl., 12 (1989), 169-179.

[6] Nevanlinna, R., Le Théorème de Plcard-Borel et al Théorie des Fonctions Méromorphes, Chelsea, New York 1974.

[7] Reinders, M., A new example of meromorphic functions sharing four values and a uniqueness theorem, Complex Variables Theory Appl., 18 (1992), 213-221.

[8] Steinmetz, N., A uniqueness theorem for three meromorphic functions, Ann. Acad. Sci. Fenn. Ser. AI Math., 13 (1988), 93-110.

[9] UEDA, H., Unicity theorems for meromorphic or entire functions, II, Kodai Math. J., 6 No. 1 (1983), 26-36.

Department of Mathematics

Daido Institute of TEChNology

DAIDO, Minami, NAgOYA 457

JAPAN 